\title{
Enquête
}

Archives de la revue Enquête

3| 1996

Interpréter, Surinterpréter

\section{L'interprétation, source de la compréhension chez Max Weber}

Interpretation, source of understanding in Max Weber's writings

\section{François-André Isambert}

\section{(2) OpenEdition}

Journals

Édition électronique

URL : http://journals.openedition.org/enquete/423

DOI : 10.4000 /enquete.423

ISSN : 1953-809X

Éditeur :

Cercom, Éditions Parenthèses

Édition imprimée

Date de publication : 1 novembre 1996

Pagination : 129-151

Référence électronique

François-André Isambert, «L'interprétation, source de la compréhension chez Max Weber », Enquête [En ligne], $3 \mid$ 1996, mis en ligne le 11 juillet 2013, consulté le 02 mai 2019. URL : http://

journals.openedition.org/enquete/423; DOI : 10.4000/enquete.423

Ce document a été généré automatiquement le 2 mai 2019. 


\title{
L'interprétation, source de la compréhension chez Max Weber
}

\author{
Interpretation, source of understanding in Max Weber's writings
}

\author{
François-André Isambert
}

1 On a relu cent fois, sans s'y arrêter, ce début d'Économie et société, définissant la sociologie comme une science qui cherche à « comprendre en interprétant » (deutend verstehen ${ }^{1}$ ) la vie sociale. Habitués que nous sommes à lier interprétation et compréhension, nous ne remarquons même plus deutend qui nous paraît redondant. Et pourtant, Weber insiste si souvent dans son œuvre sur l'interprétation qu'une négligence de style est ici exclue. Ainsi, dans sa Verstehende Soziologie (1913), première esquisse du chapitre initial d' Économie et société, on trouve, dès la première page, que «ce qui n'appartient qu'au comportement humain, c'est l'ensemble des connexions et des régularités dont le déroulement est interprétable de manière compréhensible ». Et un peu plus loin, Weber se réfère à la « compréhension (Verstandnis) conquise par l'interprétation ${ }^{2}$ ".

On peut dès lors se demander si le participe deutend spécifie la notion de verstehen qui, sans autre précision, s'étendrait depuis l'intuition affective jusqu'à la compréhension intellectuelle (Verstand désigne chez Kant l'entendement). On ferait alors fausse route, car Weber emploie le mot «interprétation» dans au moins autant de sens que « compréhension». Et pourtant, le besoin d'une telle notion est chez lui constant. D'une part, il ne peut concevoir une compréhension en quelque sorte offerte à la pensée : il faut que cette compréhension soit le résultat d'une démarche active, d'un travail intellectuel. D'autre part, elle permet de situer Weber dans la "querelle des méthodes » (Methodenstreit), où règne la plus grande confusion concernant les critères de différenciation dans une échelle de sciences allant depuis les plus empiriques des sciences de la nature jusqu'à l'histoire qui occupe le pôle opposé. De toute manière, l'interprétation se glisse comme une médiation nécessaire entre le sujet comprenant et l'objet compris. Cette discussion, en faisant l'inventaire des acceptions du terme «interprétation", permet en même temps de débrouiller cet écheveau et d'éviter quelques équivoques. 
Weber veut préciser une démarche originale pour ce qu'il appelle «sciences de la culture » sans pour autant renoncer à l'idéal d'objectivité. Bien que cet idéal ne soit pas toujours atteint dans une compréhension qui reste le plus souvent approximative, il peut être approché d'un côté par une saisie empirique sur laquelle vient se greffer l'interprétation, de l'autre par l'introduction de la rationalité au sein même de l'interprétation. Mais, une fois effectué ce rappel des exigences fondamentales du wébérianisme, le principal reste à faire, à savoir débrouiller la manière dont Weber répond à ces exigences à première vue contradictoires. Sa démarche étant d'abord hésitante et son élaboration conceptuelle progressive, nous ne pourrons nous aussi procéder que par approximations successives. Dans cette progression, l'étude sur Roscher et Knies ${ }^{3}$ occupe la première place, non seulement dans le temps mais encore par la minutie avec laquelle Weber y distingue les diverses sortes d'interprétation et leur rôle possible dans la connaissance.

\section{L'interprétation dans les sciences humaines}

\section{Roscher et l'interprétation historique}

«Roscher und Knies» fut le premier travail de critique méthodologique et théorique de Weber. Les deux dates de 1903 et 1906 qui situent la publication des deux parties de l'ouvrage, finalement inachevé, illustrent la peine qu'eut Weber, au sortir de sa grande crise, pour mener à bien cette œuvre qui fixait sa position dans l'univers intellectuel de l'époque.

5 Publiée avec une multitude de notes additionnelles, elle marque la difficulté qu'eut Weber à se dégager des alternatives créées par le débat dont les sciences sociales étaient le théâtre ${ }^{4}$. C'est beaucoup plus aisément qu'en 1904 il fit paraitre un manifeste sur l'objectivité dans ces sciences, destiné à donner son orientation épistémologique à l'Archiv für Sozialwissenschaft, et où il développait en toute liberté sa conception de la démarche historico-sociologique. Des piliers incontournables de la «méthode historique » en économie sociale, Roscher, Knies et Hildebrand, il ne retient que les deux premiers ${ }^{5}$ et fait une place toute particulière à Roscher, pionnier de la méthode historique en science économique.

6 Une première distinction lui est fournie par Roscher qui dissocie de la conceptualisation classique une forme de connaissance qui constitue une première esquisse de l'interprétation: Roscher oppose deux manières de traiter scientifiquement le réel, qu'il désigne comme " philosophique » et " historique » : d'un côté la saisie conceptuelle dans la voie de l'abstraction généralisante en éliminant les «accidents » du réel, de l'autre la restitution du réel en le décrivant dans sa réalité intégrale ${ }^{6}$.

7 Le caractère abstrait de la démarche généralisante est trop évident pour qu'on s'appesantisse sur la critique qu'en fait Weber. Ce qui nous intéresse, c'est le réalisme individualisant dont, après Rickert, il fait son profit. Or, sur ce point, la pensée de Roscher conduit à une conception qui va beaucoup plus loin qu'une simple description puisqu'il s'agit de «la connaissance de ces composantes de la réalité qui, pour nous, dans leur singularité individuelle et, eu égard à celle-ci, sont essentielles ${ }^{7} »$. Weber pénètre là dans le redoutable problème du concept d'individualité, que Roscher n'avait pas craint d'aborder. Une première entrée serait celle des relations universelles qui peuvent s'incarner dans un «individu» (être, chose ou ensemble - un peuple par exemple - doué d'une identité 
propre), considéré alors comme une combinaison de prédicats génériques. Mais, au générique s'oppose le spécifique qui se caractérise par la différenciation, atteignant son stade ultime dans l'individu. Ces relations sont capitales pour en arriver au «concept de chose individuelle ${ }^{8} »$. Weber éprouve le besoin de préciser que l'usage ayant rendu cette alliance de termes insolite, il faut s'obliger à entendre par «concept» tout élément logique ayant pour but la «connaissance de l'essentiel » : il en donne comme exemple la personne même de Bismarck ${ }^{9}$. À ce niveau, il rencontre un axiome auquel il s'attachera constamment, à savoir qu'il n'y a de réalité qu'individuelle : c'est la seconde entrée dans la notion de concept individuel.

8 Sur ce point, Weber, comme Roscher, ne pouvait que rencontrer le « concept » entendu au sens hégélien ${ }^{10}$. L'attitude ambiguë que Weber reproche à Roscher à l'égard de Hegel tient au fait que, selon lui, Roscher n'a pas exactement évalué le rapport entre la conceptualisation dans les sciences génériques et ce qu'elle devient dans la démarche historique, d'où le hiatus irrationalis ${ }^{11}$ qu'il est amené à creuser entre concept et réalité. Weber serait plus proche de Hegel, à ceci près qu'il ne pense pas que la conceptualisation du réel puisse aller jusqu'à sa déductibilité. Par ses réquisits implicites, Weber esquisse donc un cheminement de pensée qu'il ne nomme pas encore, mais qui conduit à la compréhension de l'individualité.

Or le critère de différenciation entre sciences génériques ou nomologiques et sciences de l'individuel croise celui qui sépare sciences de la nature et sciences humaines d'une manière qui fait confondre facilement les deux paires en une seule et même dichotomie. Weber fait remarquer ${ }^{12}$ que la formulation exacte de cette dichotomie, fournie par Dilthey et Simmel, avait auparavant été « esquissée » par Windelband, puis précisée par Rickert. Ce sera pour lui l'occasion de marquer à la fois son accord approximatif et provisoire avec des oppositions comme «sciences de la nature et sciences de l'esprit» ou «sciences nomologiques et sciences historiques ${ }^{13}$ » et, en même temps, son adhésion, plus spécifique, à la position de Rickert, qui fait de l'individualité de leur objet le propre des sciences historiques. Weber se verra obligé de revenir sur la diversité des critères utilisés par les divers auteurs et sur leur validité respective. De toute manière, Weber tient pour essentiel que «le cours de l'action humaine et de l'expression humaine est chaque fois accessible à une interprétation comportant un sens [sinnvollen Deutung] ${ }^{14} »$. Et Weber insiste sur le fait que c'est cette interprétation qui fait des sciences humaines un "groupe particulier ». Dès l'abord il présente l'interprétation comme recherche d'un sens, propriété que seule l'activité humaine possède. Mais il ne peut en rester là. Il recourt à Knies et à toute la mouvance irrationaliste pour trouver un nouveau point d'appui qui va être en même temps un repoussoir.

\section{Knies et la subjectivité}

10 Knies, le deuxième pilier de l'« école historique ", avait publié en 1853 Die politische Ekonomie vom Standpunkt der geschichtlichen Methode. Pour lui, les lois de la nature soumettent celle-ci au déterminisme, et il en est de même des lois économiques. Face à cela, l'action humaine affirme sa liberté, ce en quoi, selon Knies, elle est irrationnelle ${ }^{15}$. Par là, on fait entendre qu'elle ne peut être expliquée et échappe à la causalité. Retombet-on alors sur l'idée de "créativité » introduite par Wundt au sujet de la "synthèse créatrice » que la personnalité induirait dans les états de conscience? Weber discute longuement cette notion et n'a pas de mal à mettre en évidence ses contradictions. 
11 L'idée forte sur laquelle il s'appuie, c'est le fait que les comportements humains présentent une prévisibilité au moins aussi forte - et parfois plus - que les phénomènes de la nature. L'exemple qu'il fournit mérite d'entrer dans la collection des images philosophiques célèbres.

«Lorsque la tempête a arraché un bloc d'une paroi rocheuse et l'a fait éclater en nombreux débris éparpillés au sol [...] en combien de morceaux de quelles formes, le bloc a éclaté et comment, dans leur éparpillement, ils gisent regroupés [...] cela, notre savoir nomologique ne le comprend que comme n'entrant pas en contradiction directe avec lui. En revanche, une "régression" causale réelle ne serait pas seulement tout à fait impossible par suite du caractère incalculable de cet aspect du phénomène [...] mais aussi, indépendamment de cela, cette recherche causale serait sans objet ${ }^{16}$.»

Une telle indétermination contraste avec la prévisibilité attendue des actions humaines : celle d'un commandement militaire, d'une peine judiciaire, d'une expression dans nos relations avec autrui et dont on peut prévoir l'effet dans une situation donnée. En somme, Weber conçoit les phénomènes naturels comme une poussière de petits faits insignifiants que seul notre esprit rassemble pour en faire des objets en mouvement. L'essentiel des faits massifs n'est donc pas d'être soumis à la loi des grands nombres, mais de retenir notre attention, ce qui peut être le cas du regroupement de phénomènes élémentaires. Mais l'attention du savant peut aussi se porter sur d'autres types de faits. Par exemple, en biologie, un concept comme celui d'" adaptation", non répétitif mais soumis à la phylogenèse, échappe de ce fait aux explications de type nomologique et doit être soumis à exégèse ${ }^{17}$. Il en sera de même pour les faits qu'étudient les sciences sociales. Ces faits sont choisis « dans l'infinité dépourvue de sens du devenir universel ${ }^{18}$ ».

Or, sur cette voie, on rencontre la position de tous ceux pour qui, avec Knies, le sens est strictement subjectif et totalement étranger aux catégories de l'explication objective. Ainsi, pour Münsterberg, la connaissance par interprétation va-t-elle être une connaissance "subjectivante" (subjektivierende ${ }^{19}$ ), c'est-à-dire non seulement une vue subjective mais une démarche qui nous conduit à une appréhension subjective des choses. Weber n'interdit pas d'employer « interprétation » dans ce sens, mais il s'élève contre la prétention de Münsterberg d'exclure cette voie pour les sciences qui visent par ailleurs l'objectivité et de dresser l'une contre l'autre "sciences subjectivantes» et «sciences objectivantes ». L'histoire la plus attachée à l'objectivité peut avoir recours à l'intuition concrète (Veranschaulichung ${ }^{20}$ ) pour communiquer une expérience affectivement vécue (Gefühlserlebnis) ou le caractère global d'une culture.

14 On ne doit jamais oublier que, d'un point de vue scientifique, l'interprétation est avant tout explicative et répond à une "exigence de causalité » (kausales Bedürfnis). Le comportement humain se prête particulièrement à l'interprétation de deux manières distinctes, quoique très proches l'une de l'autre. Cela peut consister à

"déterminer l'objectif [d'un acte] et cela pas seulement en tant que "possible" au sens où il s'agirait de le rendre "conceptuellement" compatible avec notre savoir nomologique, mais de le "comprendre" c'est-à-dire de trouver un "motif" concret, "intérieur", "susceptible d'être revécu" (nacherlebbares) ou un ensemble de motifs ${ }^{21}$ ".

15 Ainsi interprétable, le comportement humain est moins irrationnel qu'un fait naturel singulier, car il y a davantage dans cette interprétation que dans la simple prévision inductive (au cas où un fait n'est pas directement compréhensible, nous nous rabattons sur les lois de la psychologie et surtout de la psychopathologie ${ }^{22}$ ). Ou bien, nous plaçant 
sur un plan logique, nous pouvons insister sur la différence par rapport à la simple observation des régularités naturelles. "Nous demandons à l'interprétation le "sens" de l'action ${ }^{23}$. " Celui-ci ne nous est pas donné par une observation empirique des lois du comportement, qui ne nous dévoile pas le "pourquoi» (warum ${ }^{24}$ ) de la constance des réactions observées. Même si nous ne savons pas ce que signifie le «sens", nous savons qu'il réside dans ce « pourquoi » qui comble un vide de la connaissance.

Ce qu'on peut appeler « interprétation rationnelle » (rationale Deutung ${ }^{25}$ ) touche de près à la précédente. Elle repose sur l'hypothèse selon laquelle l'action à expliquer a été pensée comme un rapport de moyens à fins. Hypothèse correspondant parfois à la réalité, mais pouvant aussi consister à donner un sens clair et simplifié à une conduite dont la motivation pouvait être obscure et complexe. En ce cas, l'interprétation est une véritable construction, résultat d'une rationalisation a posteriori, l'édification d'une sorte de «modèle» qui permet de trouver un fil conducteur dans une suite d'événements obscurément orientés vers une fin et sans lequel on ne rencontrerait qu'absurdité. Ce genre de modèle va resurgir lorsque nous évoquerons les «types idéaux ».

17 Enfin - et c'est là qu'on trouve le point de vue le plus originalement wébérien - c'est encore une forme d'interprétation, celle que nous nommerons « exégèse axiologique » ou, plus littéralement « interprétation qui relie aux valeurs » (wertbeziehende Interpretation ${ }^{26}$ ). Processus complexe, nettement distinct de toutes les autres formes d'interprétation, elle joint, comme nous aurons l'occasion de le voir de plus près, l'intuition des valeurs et l'objectivité des constats; elle possède une fonction essentielle dans le choix de l'objet d'étude en évaluant ce qui vaut la peine d'être étudié.

\section{Médiations}

\section{Concepts}

18 Le concept est l'objet lui-même en tant qu'il est conçu. L'importance de cette maxime a été soulignée dans le cas du concept d'un objet historique. Nous sommes bien obligés, après un premier tour d'horizon, d'avouer que cette maxime est seulement normative. Le concept, même s'il fournit l'essentiel de l'objet, ne peut donner qu'une approximation dont sont exclues toutes les particularités de l'objet concret. Il est, nous l'avons vu, des interprétations purement affectives. Les interprétations intellectuelles, les seules qui intéressent directement la science, sont obligées d'avoir recours aux concepts qui, pour autant qu'ils gardent une distance - si faible soit-elle - avec l'objet, n'en constituent pas moins une étape intermédiaire, médiation obligée dans une vision indéfinie du concret. Mais, inversement, le concept, si imparfait soit-il, contribue à constituer l'objet dans une vision du monde qui, comme celle de Weber, rencontre le réel comme une poussière amorphe au sein de laquelle notre pensée a aggloméré des formes.

La tradition aristotélicienne accorde au concept une nature générique ${ }^{27}$. En introduisant la notion de type idéal, Weber voit plus large. Il est faux, comme on le fait parfois, d'opposer " concept générique » et " type idéal », même si Weber nous donne un mode de construction du type idéal qui n'a apparemment rien de générique et s'apparenterait plutôt à la caricature ou à la «stylisation ». C'est en accentuant un ou plusieurs traits de l'objet et non en sélectionnant les traits communs à un genre qu'on campe en général un type idéal (et on voit déjà ici comment la valeur peut intervenir). Mais ce croquis peut aussi bien être celui d'un individu (comme Bismark) que celui d'un genre comme celui 
d'artisanat ${ }^{28}$. Seulement, il n'est pas dit que les traits retenus pour notre tableau de l'artisanat s'obtiendront par superposition de tous les artisans possibles. C'est en retenant les traits les plus significatifs à nos yeux que nous faisons habituellement notre tri. Si les traits retenus sont ceux qui sont communs au plus grand nombre d'artisans, notre type idéal coïncidera avec le type générique. Mais le plus souvent, notre choix sera guidé par une idée de valeur.

Toutefois, si Weber utilise l'expression "type idéal» avec une grande souplesse, en admettant sous ce nom tout «tableau de pensée » (Gedankenbild) pourvu qu'il présente une unité, tout en sachant qu'il s'écarte des données empiriques et représente une " utopie ${ }^{29}$ ", sa préoccupation constante est double : d'une part donner au type idéal un caractère "génétique ", c'est-à-dire pouvoir construire sur cette idée de base un édifice suffisamment riche, d'autre part identifier les relations causales ou finales, de ressemblance ou de différence, entre le type idéal et l'objet empirique auquel il se réfère. Cette double préoccupation fait du type idéal le médiateur par excellence de l'interprétation. Certes, le type idéal est une conception et non un jugement - et toute interprétation comporte un jugement - mais chaque élément d'un jugement, sujet comme prédicat, peut être idéaltypique, de même que la totalité du système tissé entre ces éléments. L'interprétation scientifique proprement dite consiste en de multiples essais comprenant la conception des types idéaux, puis la tentative de jugement (par exemple de causalité) qui lie ces types entre eux, puis la confrontation avec les données empiriques pertinemment choisies, enfin l'acceptation ou le rejet de l'hypothèse.

21 Apparentés aux types idéaux sont les " points » (Gesichtspunkten ${ }^{30}$ ), tel que le point de vue économique dans l'analyse sociologique. Comme le type idéal il choisit certains traits pour les mettre en relief, mais c'est tout un pan de la vie sociale qui est alors privilégié. L'« interprétation économique de la réalité » (die ökonomische Deutung der Wirklichkeit ${ }^{31}$ ) est très exactement un point de vue déterminé sur les faits sociaux.

«Libérés de la croyance surannée selon laquelle l'ensemble des phénomènes culturels peut être déduit, comme produit ou comme fonction, de constellations d'intérêts matériels, nous croyons pour notre part qu'une analyse des phénomènes sociaux et des processus culturels menée du point de vue spécifique de leur conditionnement et de leur portée économiques a constitué un principe scientifique d'une créativité féconde et que son emploi prudent et libre de préjugés dogmatiques le reste encore dans l'avenir. Ce qu'on appelle la "conception matérialiste de l'histoire" comme "vision du monde" ou comme dénominateur commun de l'explication causale de la réalité historique étant à rejeter de la manière la plus nette il ne reste que le souci d'une interprétation économique de l'histoire (ökonomischen Geschichtsinterpretation) est un des buts essentiels de notre revue $^{32}$.»

Pourvu qu'on réduise les réserves de Weber à l'égard du marxisme à leurs justes proportions ${ }^{33}$, le passage cité est à retenir. En effet, on y trouve la distinction entre un économisme dogmatique et global et une interprétation économique qui ne vise pas à être autre chose qu'un éclairage particulier sur l'histoire sociale. Cette interprétation "particulière " est féconde, sans pour autant barrer la route à des interprétations fournissant des éclairages différents. C'est ce que Weber était en train de pratiquer au moment où il écrivait ces lignes ${ }^{34}$, en réfléchissant sur les rapports entre vie économique et religions. L'« esprit du capitalisme » est éclairé par l'éthique protestante, alors que la religiosité propre à telle ou telle couche sociale est éclairée par sa situation économique. 


\section{Valeurs} impliquant des valeurs.

«Toute connaissance de la réalité culturelle se présente toujours comme une connaissance acquise d'un point de vue spécifique particulier. Lorsque nous exigeons de l'historien et du sociologue comme condition élémentaire qu'ils puissent distinguer ce qui est important et ce qui est sans importance et que, pour cette distinction, il possède le "point de vue historique" requis, cela veut dire seulement qu'il doit comprendre comment installer les processus de la réalité consciemment ou inconsciemment - sur le plan des "valeurs culturelles" universelles et, d'après cela, établir des relations qui soient pour nous interprétables ${ }^{35}$.»

Il ne faut pas confondre, comme on l'a vu plus haut, l'interprétation subjective-affective (subjektive getfühlmäszige) et l'«interprétation liée aux valeurs» (wertbeziehende Interpretation). Expression curieuse à première vue, l'interprétation relevant apparemment du seul domaine de la connaissance et non de la valeur. Mais cette interprétation ne peut éviter d'avoir à connaître d'« objets historiques » axiologiquement qualifiés. Weber va longuement développer cette proposition dans ses « Études critiques ${ }^{36}$ ». Il y discute en effet avec déférence l'ouvrage d'Edward Meyer, Zur Theorie und Methodik der Geschichte. Il se montre d'accord sur l'essentiel de la méthode et sur son retour à l'individu. Mais Meyer va plus loin. Au sein de la réalité, c'est ce qui est effectif (wirksam) qui doit être retenu, c'est-à-dire ce qui possède une importance causale, une efficience. Mais aux yeux de Weber, ce critère n'est pas absolument décisif. Si un choix est encore nécessaire, il se fera au nom de l'«intérêt historique ", expression qui demande à être élucidée.

Weber prend et retourne dans tous les sens le cas des lettres de Goethe à Madame de Stein $^{37}$. Ces lettres semblent avoir exercé sur Weber une véritable fascination, représentant le type le plus pur de ce que l'historien sociologue peut se donner comme objet valable en dehors de toute considération d'action causale sur le devenir des choses. On aurait attendu qu'il en fasse lui-même l'analyse textuelle. Il nous donne en fait un texte long et embrouillé, mais où il creuse autant qu'il est possible la ligne-frontière entre la connaissance et la valeur. On "interprétera » ces lettres comme une expression des sentiments éprouvés par Goethe au cours de cette période de sa vie. Il en émerge alors plusieurs couches de sens possibles.

On peut tout d'abord les voir comme des chainons dans le développement de la personnalité de Goethe. L'alliance de la passion et de l'ascétisme dont elles témoignent a sans doute influé sur la production littéraire ultérieure de leur auteur et peut leur conférer à ce titre la qualité d'« historiques ». Mais, ajoute Weber, même si on supposait que ces lettres n'eussent pas laissé de trace dans l'œuvre postérieure de Goethe, elles pourraient apporter quelque chose d'essentiel sur Goethe, en nous dévoilant un peu de la conduite de sa vie et contribueraient alors à l'explication de son personnage historique. Supposons ensuite qu'au lieu de dévoiler la personnalité originale de l'homme-Goethe, elles campent un type caractéristique de son milieu, de sa nation, ou de son époque. Ce type pourrait avoir encore une fonction explicative, dans la mesure où les individus sont influencés par le «type social » qui sert d'idéal et de modèle à leur société et leur milieu, ou encore parce que certaines conditions sociales provoquent généralement ce type de 
conduite. On pourrait enfin concevoir que rien de tout cela ne puisse nous retenir et que seule puisse nous attirer la connaissance pure, du fait de notre spécialité (par exemple la psychiatrie) ou de notre désir de connaître, encouragé par la conviction vague que toutes les connaissances finissent par converger.

Dans tous ces cas, et de moins en moins à mesure que nous avancions, les fameuses lettres ne nous auraient servi à expliquer quelque phénomène que ce soit. C'est même, jusqu'à un certain point en ce sens qu'elles sont, pour nous, «significatives ». Mais plus leur utilité est mise en question, plus se dessine en filigrane quelque chose d'irréductible ${ }^{38}$ :

«Le contenu de ces lettres, pour autant que nous ne regardons pas vers quelque particularité externe ne résidant pas dans l'espace délimité des "traits significatifs" en eux-mêmes, est pour nous, dans son unicité, un objet d'estimation et il en irait encore ainsi même si nous ne connaissions absolument rien de leur auteur ${ }^{39}$.»

Tous ces degrés de "significativité » sont dépassés par le plus haut d'entre eux. Ces expériences peuvent " être significatives " pour nous, et pas seulement comme « causes " ou comme "moyens de connaissance ». Notre intérêt est double: d'une part nous nous attachons à ce que l'objet a d'unique en lui-même, d'autre part celui-ci donne lieu à réflexion et méditation. Mais cette bipartition ne doit pas être confondue avec une autre que le lecteur a du mal à dégager parce que plusieurs bifurcations secondaires s'enchaînent dans les pages qui suivent.

Une fois mentionnée la distinction entre l'attention pour l'objet lui-même et le même objet comme source de méditation, vient la distinction majeure :

"Cette "exégèse" ou, comme on dit, cette "interprétation" peut maintenant emprunter de fait deux directions nettement séparées logiquement, mais presque toujours confondues. Elle peut être et deviendra en premier lieu une "exégèse axiologique" (Wertinterpretation), c'est-à-dire qu'elle peut nous apprendre à "comprendre" la teneur spirituelle (geistigen Gehalt) de cette correspondance et, en même temps, à déployer et élever vers la lumière d'une évaluation distincte ce que nous "ressentons" de manière obscure et confuse ${ }^{40}$."

Vient alors l'énoncé de la «direction", à ne pas confondre avec celle-là. Une telle interprétation

« n'est, à cette fin, d'aucune manière forcée elle-même de produire un jugement de valeur ni de le suggérer [...]. À l'égard du comportement de Goethe et de Madame de Stein, le moderne et détestable censeur des choses du sexe (Sexualbanause) comme aussi le moraliste catholique, même s'ils se montrent d'abord "compréhensifs", auront pour attitude essentielle de s'en détourner. » dans l'œuvre wébérienne. «La différence n'est pas seulement entre évaluation (Wertung) et rapport aux valeurs (Wertbeziehung), mais aussi entre évaluation et exégèse axiologique ${ }^{41}$.» Cette manière de pousser le plus loin possible la différenciation s'explique dans la mesure où Weber veut bien faire entendre qu'il ne faut pas seulement éviter de confondre jugement de valeur et mise en relation avec des valeurs, mais que l'exégèse des valeurs, concept qu'il a lui-même introduit, pourrait être confondue avec une évaluation parce que les deux démarches sont proches l'une de l'autre. L'une et l'autre proviennent d'un même "intérêt pour les valeurs" (Wertinteresse) et toutes deux débouchent sur une estimation. Mais les valeurs de référence de la seconde n'expriment pas une préférence personnelle du savant : elles sont d'ordre culturel (voire transculturel).

31 Weber accumule les exemples d'objets d'estimations possibles: le Capital de Marx, ou Faust, le plafond de la Chapelle Sixtine, les Confessions de Rousseau, les pensées de sainte 
Thérèse d'Avilla, de Madame Roland, de Tolstoï, de Rabelais, de Marie Bashkirtseff ou le Sermon sur la montagne. Tous ces objets culturels peuvent se voir appliquer des jugements de valeur extrêmement diversifiés qui reposent sur des interprétations de leur sens. Ces estimations sont subjectives et non scientifiques. Elles restent malgré tout des exégèses axiologiques, dans la mesure où l'expertise enrichit et affine la connaissance. Weber en soutient l'originalité.

«Puisque cette sorte d'interprétation n'est dirigée ni vers la recherche de faits causalement pertinents pour une relation historique, ni vers l'abstraction d'éléments typiques, valables pour la construction d'un concept générique, mais qu'en contrepartie elle conçoit plutôt ses objets "à leur guise" et "pour eux-mêmes" (au sens où Meyer parle de la "totalité d'une culture" dans son unité, par exemple celle de la civilisation grecque lors de son épanouissement) et qu'elle fait prendre conscience du rapport aux valeurs, elle n'entre dans aucune des catégories de la connaissance dont les relations directes ou indirectes avec la notion d'"historique" ont été mentionnées ${ }^{42}$. »

Ce n'est pas qu'il faille larguer les amarres avec l'histoire. Il s'agit, en fin de compte, de comprendre des faits historiques, mais c'est seulement après en avoir saisi le sens, puis en avoir dégagé l'importance significative, qu'on peut mettre en lumière leur dynamique. La pensée la plus intime de Goethe est pénétrée par l'action du milieu dans lequel il vivait et par les événements qui se déroulaient autour de lui. Weber est ici obligé de se défendre contre la tendance de Meyer à placer l'exégèse axiologique hors du devenir qui serait l'apanage de l'histoire: la première serait intemporelle, tandis que seule l'histoire s'écoulerait dans le temps. On retrouve donc une dualité entre l'importance causale et l' importance axiologique. Mais Weber, qui s'est surtout efforcé jusqu'ici de les séparer, va désormais les traiter comme complémentaires.

\section{L'interprétation au service de la connaissance}

\section{À la recherche de la cause}

C'est une nouvelle fois de compréhension interprétative ${ }^{43}$ qu'il s'agit lorsque nous cherchons, en histoire, la vraie cause d'un événement. Particulièrement exemplaire est la recherche de la cause du déclenchement de la révolution de 1848 à Berlin, que certains attribuent à deux coups de feu tirés inopinément.

«Si on avait rendu plausible que, sans ces deux coups de feu devant le château de Berlin, une révolution aurait pu, selon les lois générales de l'expérience, être évitée, et cela avec une dose décisive de vraisemblance, parce que, de façon démontrable, la combinaison des autres conditions, sans l'intervention de ces coups de feu n'aurait pas, ou n'aurait pas considérablement "favorisé" (begüngstigt) [...] une révolution, toujours selon les lois générales de l'expérience, il aurait alors fallu parler d'un fait provoqué "accidentellement" (zufällig) et même attribuer comme cause à la révolution de mars seulement ces deux coups de feu, chose véritablement difficile à penser ${ }^{44}$.»

L'insatisfaction laissée par ce genre d'explication montre bien que nous sommes à la recherche d'une cause d'un autre ordre. L'allemand aide à cela en distinguant Kausalität et Ursächlichkeit. Bien que ces deux termes soient à la rigueur interchangeables, leur dualité peut servir à mettre en évidence, dans le terme Ursäche, la cause fondamentale, laissant souvent - et Weber use volontiers de cette possibilité - à l'adjectif kausal le soin de qualifier la simple antécédence empirique. Le même genre d'insatisfaction assaille le 
lecteur lorsque l'historien attribue une guerre à la décision d'un homme d'État, Hannibal, Frédéric le Grand ou Bismarck ${ }^{45}$.

Certes, Weber ne considère pas comme « oiseuse » (mübig) la question des conséquences de telles décisions. Mais on a eu tort, en s'appuyant sur le cas de l'importance causale décisive de la bataille de Marathon ${ }^{46}$, d'attribuer en propre à Weber la «méthode » que l'on pourrait dire de l'« histoire-fiction", qui consiste à imaginer les conséquences de l'absence d'un fait historique pour en éprouver l'importance causale. Pour Weber, cette manière de faire est spontanée et les historiens ne s'en privent pas. Il lui reconnaît donc une certaine validité. C'est pourquoi, finalement, dans le cas des deux coups de feu de Berlin, il ne leur dénie pas toute efficience, parmi la multitude des conditions de réalisation de la révolution allemande de mars 1848 . Mais là n'est pas la question essentielle. Tant que nous en sommes à dénombrer les «causes» (au sens kausal) nous restons sur le plan de nos habitudes de pensée empirique (Weber insiste sur l'expression "selon les lois générales de l'expérience »). Or ce qui compte, c'est autre chose, c'est pour emprunter un terme aux juristes - la « causalité adéquate » (adäquate Verursachung ${ }^{47}$ ).

Bien que le texte s'interrompe au moment où Weber va définir la causalité adéquate thème sur lequel il reviendra, mais sans en donner de définition complète -, nous pouvons dès maintenant l'apercevoir en quelque sorte en creux dans la critique de ce qui est donné comme son opposé, la " causalité accidentelle ${ }^{48}$ ». Cette critique, Weber avoue la devoir pour l'essentiel à von Kries qui évoque la question de la causalité humaine à propos de la responsabilité criminelle ${ }^{49}$. Dans les deux cas, ce qui est recherché, ce n'est pas la «combinaison de lois» (Gesetzlichkeit) qui a abouti à un certain effet, mais le " conditionnement causal concret d'un résultat ${ }^{50}$ ». On devine immédiatement que, dans la causalité, il faudra retrouver l'«individualité » (au moins dans le sens très large que Weber confère à ce terme en histoire) et son orientation intentionnelle, ce qui nous fait rejoindre le sens recherché par toute interprétation. En revanche, le constat des régularités empiriques ne conduit qu'au probable et leur accumulation n'arrive pas à combler le fossé qui les sépare d'une causalité certaine.

Pour en revenir à la bataille de Marathon, Weber ne recommande pas de soupeser les facteurs multiples qui l'ont occasionnée et les innombrables effets qu'aurait pu avoir une victoire des Perses, mais de se demander, après avoir dégagé les « éléments essentiels ", à savoir l'affrontement de deux civilisations «par quelles opérations logiques nous effectuons l'examen - et rendons possible de le fonder sur une démonstration - selon lequel une telle relation causale entre les éléments "essentiels" du résultat et des éléments déterminés provient de l'infinité des facteurs déterminants ${ }^{51}$ ?"

Sous cet angle, nous pouvons nous livrer à la fiction historique, mais sachant bien qu'il a fallu d'abord choisir ces « éléments essentiels » et que les conséquences prévues par nos manipulations mentales ne sont rien d'autre que celles auxquelles «nous pouvons nous attendre " ( zu erwarten gewesen»). Ce sont des abstractions, des constructions invérifiables stricto sensu.

\section{Les formes d'adéquation}

Passant en revue les divers degrés de compréhensibilité des types de liaison que nous établissons entre les événements et les comportements, Weber se référera à nouveau, quelques années plus tard, à la notion de "causalité adéquate » (adäquat Verursachung) à 
laquelle il accole l'adjectif sinnhaft que traduit mal le mot "significatif » et qu'il vaut mieux rendre un peu lourdement par « du point de vue du sens ${ }^{52}$ ». L'adéquation du sens dans une relation causale se marque par le degré d'« évidence » (de la compréhension) que produit l'interprétation que l'on propose. Aussi est-ce bien le même type qui possède la plus forte adéquation de sens et le plus haut degré d'évidence, le type de la « rectitude » (Richtigkeittypus ${ }^{53}$ ) qui est présenté comme une forme de rationalité : ce qui nous ramène vers l'« interprétation rationnelle » que nous avons citée. Ici, la pensée de Weber devient plus difficile à démêler. En effet, s'il écrit en toutes lettres: «La coïncidence avec le type de la rectitude est la plus compréhensible parce qu'elle est la relation causale "la plus adéquate du point de vue du sens" " (Koinzidenz mit dem Richtigkeittypus ist der verständlichste, weil « sinnhaft adäquateste Kausalzusammenhang »"5), il avait donné plus haut le premier prix de compréhensibilité à l'«interprétation rationnelle par finalité ${ }^{55}$ » qu'il recommandait de ne pas confondre avec la précédente ${ }^{56}$. Cette variation dans l'ordre de préséance s'explique par une différence d'approche.

La rationalité téléologique (Zweckrationalität) se place au point de vue subjectif de l'acteur: celui-ci enchaîne-t-il bien aux fins qu'il se propose des moyens (fussent-ils magiques) qui soient intelligibles en tant que tels? Cette manière de pénétrer dans l'intention d'autrui nous fournit une vue intérieure irremplaçable ${ }^{57}$. On voit à quel point Weber est à l'opposé de ceux qui, comme Litt ou Scheler, attribuent à la communion affective le plus haut degré de pénétration de la pensée d'autrui. Les intuitions affectives (avec reconstitution du vécu) sont maintenant situées après les interprétations rationnelles dans l'ordre de l'évidence. En deçà, on trouve l'incompréhensibilité absolue de certains états affectifs que nous ne pouvons pas nous représenter, comme par exemple certains états mystiques ${ }^{58}$.

41 Mais la cohérence interne de la rationalité téléologique, dont il ne faut pas oublier que c'est nous-mêmes qui la reconstruisons comme hypothèse particulièrement limpide, peut se trouver en contradiction avec les critères contemporains communément admis. À ce type de rationalité reposant sur une relation abstraite de finalité, s'oppose le type de la «rectitude» qui a pour critère la reconnaissance qu'une démarche intellectuelle est valable dans le milieu où elle est produite. Or, la norme de cette rectitude peut être déterminée empiriquement et des facteurs de tous ordres peuvent avoir contribué à la fixer : nous n'épousons plus avec notre propre sens de la finalité ce qui est censé conduire la pensée d'un homme ou d'une collectivité selon une logique différente de la nôtre. C'est à l'histoire de la science et, plus généralement, de la logique que nous avons recours pour nous dire ce qui, en ce temps et dans ce lieu, était considéré comme rationnellement correct. Mais, du même coup, l'histoire confère à la norme une validité objective comme ayant contribué à la pensée rationnelle.

«Ainsi les énoncés et les normes mathématiques, là où ils sont l'objet d'une recherche sociologique, par exemple lorsque le degré de leur rationalité par rectitude s'applique à l'objectif d'une étude statistique ne sont à nos yeux, d'un point de vue logique, rien d'autre que les usages conventionnels d'un comportement mental, même si leur validité est par ailleurs présupposée par le travail du chercheur ${ }^{59}$."

Revenons à la décision d'un homme d'État qui déclare une guerre. La cause de la guerre va bien se trouver dans la motivation de cet homme d'État, mais il y a deux manières d'envisager cette motivation. La première, se plaçant du point de vue interne de cet individu va essayer de reconstituer ce qu'a été sa pensée à cette occasion, en tentant de reconstituer les buts qu'il poursuivait et les moyens qu'il employait. À s'en tenir à cette 
approche, on ne dépasse pas les limites de la psychologie ${ }^{60}$. On peut même faire bonne mesure en incluant dans cette motivation les traits de personnalité qui ont pesé dans la décision ${ }^{61}$. Au fond, cette rationalité téléologique est censée être universelle et peut être assimilée, pour quitter un moment l'univers conceptuel wébérien, à une faculté du psychisme humain. La seconde manière de procéder se réfère à la sociologie de deux façons : d'abord en se référant non à une rationalité intemporelle, mais à une rationalité relative à un temps et à un lieu, capable d'intégrer les valeurs culturelles de ce temps et de ce lieu, puis en faisant entrer dans la motivation toutes les circonstances dont le ou les décideurs ont dû tenir compte pour déterminer leur vouloir, ce qui revient à situer ces circonstances mêmes au rang de causes déterminantes. On voit qu'inversement la causalité des circonstances ne fait qu'une avec la motivation des hommes d'action qui ont agi sur les événements. D'où, si on veut, la rationalité relative - compte tenu d'accidents imprévisibles comme l'invasion des polders hollandais par la mer - de l'action des circonstances en histoire. Ce rapprochement ne conduit pas pour autant à une position quasi hégélienne, identifiant réel et rationnel.

\section{Adéquation causale et adéquation selon le sens}

"Ce contre quoi la sociologie s'élèverait, ce serait d'admettre que la "compréhension" et l'"explication causale" n'aient aucune relation entre elles, si vrai soit-il qu'elles commencent leur travail à des pôles complètement opposés du devenir ${ }^{62}$.»

La "compréhension" dont il s'agit ici puise son inspiration dans la recherche de la coïncidence entre deux subjectivités : elle s'applique assez bien au décryptage du sens, selon la rationalité téléologique ou l'identification affective. L'« explication causale », elle, plonge ses racines dans l'expérience des choses. Elles convergent en direction du même objectif : rendre compte causalement des faits, mais sans jamais pouvoir se rejoindre entièrement. C'est pourquoi, aiguisant le paradoxe, Weber n'hésite pas à mettre en opposition « compréhensibilité » (Verständlichkeit) et « fréquence » (Häufigkeit $\left.{ }^{63}\right)$ : ces deux approches non seulement sont sans corrélation positive, mais varient souvent de manière inverse si on admet que, comme à la Bourse, les hommes agissent souvent de manière irrationnelle. Reste que la conduite rationnelle sert de modèle à la conduite effective, dont elle constitue le type idéal; elle engendre même une chaîne de types idéaux qui est en même temps une cascade d'interprétations.

«La rationalité objective par rectitude, en regard d'une conduite empirique, la rationalité téléologique, en regard de ce qui est compris psychologiquement comme ayant un sens, et enfin ce qui est compris comme ayant un sens, en regard du comportement motivé de manière incompréhensible, servent de type idéal dans le rapprochement par lequel des éléments irrationnels (dans les divers sens du mot) causalement pertinents sont retenus à des fins d'imputation causale ${ }^{64}$. "

Cette phrase est capitale pour caractériser l'interprétation wébérienne. Elle situe exactement les rapports du compréhensible et du réel en même temps que la situation ambiguë de la causalité qui participe de l'un et de l'autre. Si l'«évidence» d'une interprétation explicative ne suffit pas à valider le lien nomologique entre plusieurs phénomènes, elle possède, dans la compréhension de l'action humaine, un pouvoir de clarification issu de la rationalité que nous introduisons dans le réel observable. Inversement, l'observation et l'établissement empirique de lois ne nous permettent aucunement de comprendre la relation entre les phénomènes, surtout si ceux-ci sont provoqués par une action humaine, mais ils nous forcent à examiner les raisons que se 
donnent les acteurs, compte tenu des valeurs qui ont cours dans leur environnement. Ainsi la rationalité (et Weber élargit volontiers ce concept à la finalité de toute action orientée) pénètre-t-elle notre aperception des choses et des comportements par le recours aux types idéaux des raisons par lesquelles nous les interprétons, « raisons » qui sont issues soit de notre propre sens de la finalité intentionnelle des actes, soit d'hypothèses au moyen desquelles nous reconstituons la «justesse» vers laquelle tendaient les personnages de la scène sociale.

C'est sans doute la position malaisée de la « rationalité par rectitude » qui amène Weber à la redistribution bien connue entre rationalité téléologique, ou selon la fin (Zweckrationalität), et axiologique, selon la valeur (Wertrationalität ${ }^{65}$ ). Il s'agit à première vue de deux types de conduite plutôt que de deux types d'interprétation. Mais on voit assez rapidement que la préoccupation propre à Max Weber de comprendre ces types de conduite le ramène vers les modes principaux de l'interprétation. Le premier type de rationalité est en continuité avec ce qui a été dit de l'orientation subjective vers un but. La Wertrationalität, on le sait ${ }^{66}$, se caractérise par une cohérence dans la soumission à certaines valeurs. La Richtigkeit (rectitude) peut être, bien que Weber ne le précise pas, absorbée par la Wertrationalität, comme cas particulier (si toutefois la traduction que nous en proposons ici est valable). N'est-elle pas l'application stricte des normes issues des valeurs de vérité portées par le milieu culturel? Toute difficulté n'est pas pour autant écartée de ce concept parce que la rationalité axiologique n'est pas sans poser des questions méthodologiques que Weber n'a sans doute pas complètement résolues.

Pourtant les deux types de rationalité relèvent d'un même processus de compréhension, Weber ayant apparemment réussi à unifier dans une même intuition intellectuelle la logique de la finalité et celle des habitudes de pensée.

« Nous comprenons de manière absolument univoque quel sens on conçoit, dans sa pensée ou dans une argumentation, quand on emploie l'expression 2 × $2=4$ ou le théorème de Pythagore, ou quand on développe un raisonnement logique "correct" [richtig] - selon nos habitudes de pensée. De même quand, de "faits d'expérience" validés comme "bien connus" et de fins déterminées pour le genre de "moyens" à employer, on tire dans sa conduite les conséquences indubitables (selon notre expérience) qui en résultent ${ }^{67}$.

Il est vraisemblable que Weber, parlant en sociologue, peut se permettre ces assimilations parce qu'ici il est culturellement de plain-pied avec les conduites à interpréter et n'a pas à reconstituer les habitudes de pensée d'une autre époque, comme l'historien ou l'anthropologue se doivent souvent de le faire. De plus, la libre circulation qu'il établit entre rationalité axiologique et téléologique profite en définitive à la rationalité par «rectitude» qui se trouve entraînée par ce mouvement et ainsi englobée dans un concept, moins cloisonné, de rationalité. Comme Weber le répète deux fois ${ }^{68}$,

« ce qui s'appelle "comprendre" c'est [...] soit "la saisie interprétative" d'une réalité historique ou d'une moyenne dans le phénomène de masse traité par le sociologue, soit un pur type idéal scientifiquement construit. Cela veut dire que, dans les deux cas, nous attribuons un sens à la conduite d'agents réels ou fictifs ${ }^{69}$. »

Mais cela ne veut pas dire qu'il ne subsiste pas de fossé entre compréhensible et réel. L'exemple souvent donné par Weber est la loi de Gresham, «interprétation rationnelle évidente" selon laquelle lorsque deux monnaies sont en compétition, "la mauvaise chasse la bonne ». Mais seule l'expérience statistique peut nous apprendre si, de fait, la conjoncture économique n'arrive pas à faire disparaître une monnaie trop faible. Dans un 
cas pareil, il vaudrait mieux commencer par l'observation, puis en tenter une interprétation ${ }^{70}$. De manière générale,

" "Adéquat selon le sens" est le qualificatif d'un comportement dont le déroulement est lié, dans la mesure où le rapport entre ses éléments est posé par nous, selon nos habitudes moyennes de penser ou de sentir, comme exprimant typiquement (nous avons l'habitude de dire "correctement") une relation ayant un sens. "Adéquat causalement" se dit au contraire d'une succession d'événements, dans la mesure où, selon les lois de l'expérience, il existe une chance pour qu'elle se déroule bien réellement de la même manière ${ }^{71}$. "

Ce qui est "adéquat » est évidemment l'interprétation de telles conduites, ou, ce qui revient au même, de telles conduites, dans notre pensée, comme objets d'interprétation. Au reste, quelques lignes plus loin, Weber conclut :

"Une interprétation causale correcte signifie que le cours extérieur et le motif se rencontrent et sont en même temps reconnus comme ayant entre eux des relations dont le sens est compréhensible ».

Weber arrive donc à formuler en une seule phrase ce qu'il avait longtemps cherché : ce qu'est l'interprétation frayant le chemin à la «compréhension explicative ». Destinée à décrypter l'efficacité de l'action humaine, cette explication est à la fois souple et exigeante.

La souplesse réside dans son aptitude à se couler dans le sens de la conduite humaine. Nous l'avons vu en effet tirer sa spécificité de l'action à laquelle elle s'applique « quand et pour autant que le ou les sujets de l'action lui associent un sens subjectif ", c'est-à-dire « intentionnel» $\left(\right.$ gemeint $\left.^{72}\right)$. Cette caractéristique permet de considérer toute conduite à interpréter comme " orientée ", c'est-à-dire finalisée dans un sens large, qui va depuis la rationalité strictement téléologique enchaînant les moyens et les fins, jusqu'à une affectivité animée par un telos moins distinct. Elle se prête de ce fait aussi bien à l'analyse d'un calcul mathématique qu'aux motivations plus ou moins claires d'un bûcheron qui abat un arbre. Et si de fait, seuls les individus peuvent avoir des intentions proprement dites, la sociologie interprétative attribue un sens typique à l'action des types sociaux qu'elle distingue du fait de leurs analogies ou de leurs fonctions ${ }^{73}$.

52 Les exigences de l'interprétation sont liées à son double lien avec le compréhensible et le réel. Le réel ne se lit généralement pas à livre ouvert. Une opération intermédiaire de décryptage sépare et relie la perception et l'intellection. Certes, Weber est le premier à nous donner des exemples de "compréhension immédiate", (aktuelles Verstehen), celle d'un homme qui abat un arbre ou qui pose « $2 \times 2=4^{74}$ ». Mais ces actes ont à être expliqués et l'interprétation, même instantanée, bâtit toujours une hypothèse sur les motifs. Or c'est ici que se trouve le piège: le motif le plus «évident" n'est pas nécessairement le motif réel ${ }^{75}$. Compte tenu de nos habitudes de penser, nous mettons en avant un motif économique: le bûcheron abat un arbre pour gagner sa vie. Mais une connaissance empirique des faits peut nous détromper: cet homme est peut-être un propriétaire terrien dans sa forêt et il abat des arbres pour se donner de l'exercice...

53 L'interprétation explicative (la seule qui retienne l'attention de Max Weber comme elle devrait être la seule à retenir l'attention de tout sociologue) demande à être constamment confrontée à l'expérience. Si on commence par celle-ci, comme le suggère par moments Weber, il faudra bien trouver un sens aux actes que nous observons, ce qui nous remet sur le chemin des hypothèses qui doivent alors se vérifier par une évidence interne. L'idéal de l'interprétation reste toujours la coïncidence exacte des deux adéquations (avec en plus la compréhension des conditions de réalisation de cette 
coïncidence). Mais ce but est rarement atteint, en sorte que l'interprétation, pour être adéquate selon le sens, gardera toujours un minimum d'incertitude et de distance au réel. Et celui-ci, bien qu'éclairé par l'interprétation, n'en sera illuminé que partiellement.

\section{NOTES}

1. Wirtschaft und Gesellscbaft, $4^{\mathrm{e}}$ éd., p. 1. Pour les textes comportant une traduction française, la référence au titre allemand indique un passage directement traduit de l'allemand par l'auteur.

2. «Über einige Kategorien der verstehende Soziologie» in Gesammelte Aufsätze zur

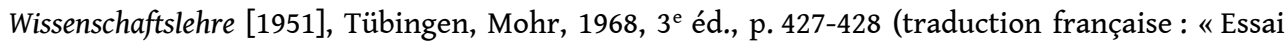
sur quelques catégories de la sociologie ", in Essais sur la théorie de la science, Paris, Plon, 1965). On notera que si verstehen désigne l'acte de comprendre, verstandnis se réfère à la compréhension acquise.

3. "Roscher und Knies und die logischen Probleme der historischen Nationalökonomie ", in Gesammelte Aufsätze zur Wissenschaftslehre, op. cit., p. 1-145.

4. Marianne Weber, Max Weber, Ein Lebensbild, Tübingen, Mohr, 1926, p. 319.

5. « Roscher und Knies », op. cit., p. 2.

6. Ibid., $1^{\text {re }}$ partie, "Roschers "historische Methode" ", p. 3. Roscher avait, dès 1842, écrit son Leben, Werk und Zeitalter des Thukydides, et, l'année suivante, Grundriss zu Vorlesungen über die Staatswirtschaft, nach geschichtlicher Methode. En 1857 était paru le System der Volkswirtschaft.

7. Ibid., p. 5.

8. Ibid., p. 6.

9. Ibid., p. 6, note 8 .

10. Ibid., p. 15-16.

11. Ibid., p. 35.

12. Ibid., p. 12.

13. Ibid.

14. Ibid., p. 13.

15. Ibid., $2^{\mathrm{e}}$ partie, « Knies und das Irrationalitätsproblem », p. 44.

16. Ibid., p. 65-66.

17. Ibid. Weber utilise alternativement le mot courant Deutung et le mot savant Interpretation avec un sens analogue. Nous utiliserons « exégèse » chaque fois que, pour Weber, Interpretation semble avoir été choisi à dessein (par exemple lorsque le terme est souligné) pour désigner une analyse plus approfondie que l'interprétation ordinaire.

18. «Die "Objektivität" sozialwissenschaftlicher und sozialpolitischer Erkenntnis", in Wissenschaftslehre, p. 180.

19. Ibid., p. 75.

20. Ibid., p. 121.

21. Ibid., p. 67. On remarquera la multiplication des guillemets, signe de ce que Weber emploie ici des expressions courantes qu'il ne prend pas entièrement à son compte.

22. Idée reprise par Jaspers (Allgemeine Psychopathologie, 1913).

23. Ibid., p. 69.

24. Ibid., p.70. Le warum allemand s'intéresse plus à la finalité de l'acte que le "pourquoi » français. 
25. Ibid., p. 126.

26. Ibid., p. 122.

27. Ibid., p. 194.

28. Ibid., p. 202.

29. Ibid., p. 191.

30. « Die "Objektivität" sozialwissenschaftlicher und sozialpolitischer Erkenntnis », op. cit., p. 146

sq.

31. Ibid., p. 168.

32. Ibid., p. 166-167.

33. Réserves accentuées par la traduction de Julien Freund (Essais sur la théorie de la science, op. cit., p. 147). En fait nous verrons Weber mettre le Capital sur le même pied que les œuvres les plus sublimes.

34. La première édition de l'Éthique protestante est de 1905.

35. « Die "Objektivität" sozialwissenschaftlicher und sozialpolitischer Erkenntnis », op. cit., p. 181.

36. "Kritische Studien auf dem Gebiet der kulturwissenschaftlichen Logik " ("Études critiques dans le domaine de la logique des sciences de la culture », 1906), in Wissenschaftslehre, p. 215.

37. Âgé d'une trentaine d'années, et après avoir publié Werther, Goethe eut à Weimar, avec Charlotte de Stein, de quelques années son aînée, une liaison platonique et épistolaire (environ 1700 lettres écrites par lui) où s'exprime, avec une passion contenue, le sens de leur existence.

38. Pour toute cette séquence, cf. «Kritische Studien auf dem Gebiet der kulturwissenschaftlichen Logik », in Wissenschaftslehre, p. 241-244.

39. Ibid., p. 245.

40. Ibid.

41. «Gutachten zur Werturteilsdiskussion im Ausschluss des Vereins für Sozialpolitik » (1913), in E. Baumgarten, Max Weber, Werk und Person, p. 122. Phrase reprise, ainsi qu'une partie du texte dans «Der Sinn der "Wertfreiheit" der soziologischen und ökonomischen Wissenschaften " (1917), in Wissenschaftslehre, op. cit., p. 498.

42. « Kritische Studien auf dem Gebiet der kulturwissenschaftlichen Logik », op. cit., p. 248.

43. Ibid., p. 277, 282, 290. Dans la seconde partie de l'article, intitulée « Objektive Möglichkeit und adäquate Verursachung in der historischer Kausalbetrachtung" (Possibilité logique et explication adéquate dans l'analyse causale).

44. Ibid., p. 287.

45. Ibid., p. 266.

46. Ibid., p. 276-277.

47. Ibid., p. 286.

48. Ibid., p. 290.

49. Über den Begriff der objektiven Möglichkeit und einige Anwendungen desselben, Leipzig, 1888.

50. « Kritische Studien auf dem Gebiet der kulturwissenschaftlichen Logik », op. cit., p. 270.

51. Ibid., p. 273.

52. «Über einige Kategorien der verstehende Soziologie» (op. cit., p. 434), que nous traduisons : "Sur quelques catégories de la compréhension sociologique» pour éviter les connotations approbatives du terme « compréhensive ».

53. Nous traduisons par "rectitude", plus près du sens étymologique de Richtigkeit et surtout pour éviter un malentendu sur "justesse", fréquemment employé dans ce cas, et qui peut signifier l'« exactitude » (comme dans le cas d'une opération dont le résultat est « juste », malgré des erreurs de calcul).

54. Ibid., p. 434.

55. « Über einige Kategorien der verstehende Soziologie », op. cit., p. 428.

56. Ibid., p. 432.

57. Ibid., p. 430. 
58. Ibid., p. 428. L'allemand emploie Evidenz aussi bien dans les cas où nous traduirions par "certitude» que dans les cas où nous dirions «évidence». Nous n'appliquons l'adjectif «évident " que pour caractériser un objet de pensée, par exemple un axiome et non pour caractériser l'acte de pensée qui le saisit. C'est pourquoi, nous répugnons à parler d'interprétation évidente s'il s'agit de la démarche intellectuelle de l'interprétation. En revanche, «une interprétation» peut être, comme une hypothèse, le contenu-objet de la démarche d'interprétation. En ce cas, on pourra dire qu'une interprétation est plus évidente qu'une autre.

59. Ibid., p. 437. Ce « chercheur » est le sociologue et non le statisticien dont il étudie les travaux. Forscher répond à soziologischer Forschung. C'est le fait que le même chercheur puisse reconnaitre comme valables certains travaux de mathématiques, tout en traitant leur méthode comme n'importe quel objet empirique, qui est digne de remarque et non le fait que le mathématicien présuppose la validité de ses propres travaux.

60. Ibid., p. 429-430.

61. Cf. "Kritische Studien auf dem Gebiet der kulturwissenschaftlichen Logik », op. cit., p. 288-289.

62. « Über einige Kategorien der verstehende Soziologie », op. cit., p. 436.

63. Ibid., p. 437.

64. Ibid., p. 436.

65. Wirtschaft und Gesellschaft, p. 12.

66. Cf. J.-C. Passeron, "La rationalité et les types de l'action sociale chez Max Weber ", Revue européenne des sciences sociales, XXXII (98), 1994, p. 5.

67. Wirtschaft und Gesellschaft, p. 2. La forme curieuse de ce raisonnement est à rapprocher de celle que Weber emploie dans «Politik als Beruf » lorsqu'il met en garde à la fois contre les moyens et les conséquences qu'entraînent certains idéaux politiques.

68. Ibid., p. 1 et 4 .

69. Ibid., p. 4.

70. Ibid., p. 5.

71. Ibid.

72. Ibid., p. 1.

73. Cf. à ce sujet la longue discussion de Wirtschaft und Gesellschaft, p. 6-10.

74. Ibid., p. 3.

75. « Über einige Kategorien der verstehende Soziologie », op. cit., p. 427.

\section{RÉSUMÉS}

Généralement la compréhension n'est pas donnée immédiatement, mais suppose un acte intermédiaire d'interprétation, en particulier lorsqu'il s'agit de conduites humaines ayant un sens. Dans le cas de l'historien, la question revient à celle de la conceptualisation d'un fait ou d'un être singulier. Il ne faut pas en conclure que la compréhension est strictement subjective ; encore moins qu'il y ait des "sciences subjectivantes", face aux « sciences objectivantes». Mais la constitution même d'un objet historique suppose, dans la multiplicité indéfinie du devenir et des points de vue, le choix de ce qui est significatif. De même, la cause d'un événement doit être choisie comme significativement adéquate à son effet. Ce type d'interprétation exige une forme particulière d'interprétation, l'« exégèse axiologique ", qui n'est pas un jugement de valeur mais 
une estimation du rapport aux valeurs. Les types d'interprétation s'échelonnent depuis la reviviscence affective jusqu'à l'interprétation rationnelle qui peut être une construction idéaltypique de ce qu'aurait été la conduite de l'agent s'il avait agi rationnellement. Toutefois, l'interprétation est toujours prise entre deux formes d'adéquations possibles, l'adéquation selon le sens, qui peut avoir pour nous divers degrés d'évidence, et l'adéquation "causale » (empirique) qui se réfère aux lois habituelles du comportement.

In general, understanding is not immediately given but presumes an intermediary act of interpretation, particularly when meaningful human behaviours are concerned. In the case of the historian, the question comes back to that of the conceptualisation of a fact or of an uncommon being. This should not lead to the conclusion that understanding is strictly subjective; still less, that there are "subjectifying sciences" facing "objectifying sciences". But the very constitution of a historical object assumes, in the indefinite multiplicity of developments and of view points, the choice of that which is significant. In the same way, the cause of an event must be chosen as significantly adequate to its result. This kind of appraisal demands a particular form of interpretation, "axiological exegesis", that is not a value judgement but an evaluation of the relationship to values. The types of interpretation spread from affective revival onto rational interpretation which can be an ideal typical construction of the probable behaviour of the agent had he acted rationally. Nevertheless, interpretation is always caught between two forms of possible adequacy, the adequacy according to meaning, which can have different degrees of evidence for us, and "causal" (empirical) adequacy which refers to customary laws of behaviour. 